\title{
Opioid Prescription Drug Use and Expenditures in US Outpatient Physician Offices: Evidence from Two Nationally Representative Surveys
} \author{
Ball $^{8}$, Kelly Barth ${ }^{9}$, Ronit Elk ${ }^{10}$, Ronnie Horner ${ }^{1,11}$ and Charles Bennett ${ }^{2}$ \\ ${ }^{1}$ Dept. of Health Services Policy and Management, University of South Carolina, Columbia, USA \\ ${ }^{2}$ Clinical Pharmacy and Outcomes Sciences, South Carolina College of Pharmacy, Columbia, USA \\ ${ }^{3}$ Dept. of Public Health and Informatics, Jahangirnagar University, Bangladesh \\ ${ }^{4}$ Health Policy \& Management Program, University of Massachusetts, Amherst, USA \\ ${ }^{5}$ College of Social Work, University of South Carolina, Columbia, USA \\ ${ }^{6}$ Military Social Work Specialization, U.S. Army Reserves, USA \\ ${ }^{7}$ Institute for Behavioral Health, Brandeis University, Waltham, USA \\ ${ }^{8}$ South Carolina College of Pharmacy, Medical University of South Carolina, Charleston, USA \\ ${ }^{9}$ Department of Psychiatry and Behavioral Sciences, Medical University of South Carolina, Charleston, USA \\ ${ }^{10}$ College of Nursing, University of South Carolina, Columbia, USA \\ ${ }^{11}$ Institute for the Advancement of Healthcare, University of South Carolina, Columbia, USA
}

Zaina P Qureshi1 ${ }^{1,2 *}$, M Rifat Haider ${ }^{1,3}$, Rosa Rodriguez-Monguio ${ }^{4}$, Nikki R Wooten ${ }^{5,6}$, Ruslan V Nikitin ${ }^{7}$, Sarah

Submission: February 07, 2017; Published: February 22, 2017

"Correspondence Address: Zaina P Qureshi, Department of Health Services Policy and Management, Arnold School of Public Health, University of South Carolina, 915 Greene Street, Columbia, SC, 29208, Phone:(803) 777-8139; Fax: (803) 777-1836; Email: qureshiz@mailbox.sc.edu

Abstract

Background: Opioids are widely prescribed for their analgesic properties. Chronic opioid use is a persistent problem in the US. Nevertheless, little is known about its prescribing and utilization patterns and overall expenditures.

Objective: This study examined secular trends in opioid prescription drug utilization and expenditures, along with factors associated with opioid prescription drug use in US physician offices.

Methods: National Ambulatory Medical Care Survey (NAMCS) and Medical Expenditure Panel Survey (MEPS) data (2006-2010), both nationally representative surveys, were used to assess the trend, predictors of opioid prescription among US adults (more than 18 years) and the opioid-associated expenditures as a whole and borne by the patients in outpatient settings.

Results: Opioid prescription drugs use among US adults in outpatient settings, as a percentage of all prescription drugs, showed a gradual increase since 2006, leveling off in 2010. Opioid prescription drug expenditures showed an upward trend from 2009 after declining over three years. Mean out-of-pocket payments per prescription steadily declined over study period.

Limitations: Cross-sectional nature and visit based information of NAMCS do not provide the actual prevalence and the reason for opioid prescription.

Conclusions: Given the upward trend in opioid prescription drug utilization and associated expenditures, clinicians may benefit from evidence-based methods of monitoring prescription opioid use to prevent misuse, abuse, and other adverse patient outcomes.

Funding: Drs. Qureshi, Haider, Ball, Horner and Bennett's efforts are partially supported by the University of South Carolina's ASPIRE I. Dr. Wooten's effort is funded by the National Institute on Drug Abuse (K01DA037412).

Keywords: Opioids; Prescription Drugs; Ambulatory Care; Prescribing Trends; Pharmaceutical Expenditures

Abbreviations: AHRQ: Agency of Healthcare Research and Quality; CDC: Centers for Disease Control and Prevention; DHS: Department of Health and Human Services; MEPS: Medical Expenditure Panel Survey; NIDA: National Institute on Drug Abuse; NCHS: National Center for Health Statistics; NAMCS: National Ambulatory Medical Care Survey; PDMP: Prescription Drug Monitoring Programs 


\section{Introduction}

Due to the elevated potential for misuse and abuse of pain medications, particularly newer options, effective treatment for acute and chronic pain continues to be a topic of much contention among clinicians and researchers worldwide. The number of Americans suffering from pain is greater than those suffering from cancer, heart disease and diabetes combined [1]. It is estimated that about 1 in 4 Americans experience pain lasting longer than one day, and that more than 100 million U.S. residents suffering from chronic pain generate healthcare costs ranging from $\$ 560$ to $\$ 635$ billion [2].

The short-term utilization of prescription opioids to address acute pain is clinically effective and is the therapy of choice for the treatment of moderate to severe pain [3]. However, longterm prescription opioid use is associated with increased risk of misuse and abuse, which can ultimately result in overdose and death. Approximately 6.1 million Americans abuse or misuse prescription drugs resulting in about 16,000 deaths [4] and 475,000 emergency room visits annually [5]. Prescription drug abuse is second only to marijuana use in the nation and painkillers are considered a major contributor to unintended drug-related mortality [6]. These findings are supported by the National Institute on Drug Abuse (NIDA) reports indicating that not only have the sales of prescription painkillers in the US increased dramatically in the past decade, but so have the number of fatal poisonings due to pain medications [7].

Prior research has assessed trends in the number of opioid prescriptions in the U.S. using the National Ambulatory Medical Care Survey (NAMCS). These studies focused on trends or prevalence rates analysis of opioid prescriptions for (1) specific diseases or conditions (e.g., musculoskeletal pain [810], headache [11], osteoarthritis [12], non-malignant pain [1315]), (2) in different health care settings [16-18], and (3) among different population groups (adult population [19], older adults [20]). Overall, prior research showed an overtime increase in the number of opioid prescriptions in the US over the period between 1980 and 2010 .

This report extends the literature by combining the NAMCS and the Medical Expenditure Panel Survey (MEPS) data to assess secular trends in the utilization and expenditures of opioid prescription drugs in the U.S. The aims of this study were to (1) describe secular trends in opioid prescribing patterns in US outpatient settings in the period 2006 to 2010, (2) identify factors associated with opioid prescription drug utilization in physician offices and (3) estimate opioid prescription drug expenditures by sources of funding.

\section{Materials and Methods}

\section{Data Source}

Study data were derived from NAMCS for the period 20062010. The NAMCS is a nationally representative dataset on the provision and utilization of outpatient medical care services
[21]. In this study, we used physician reported data on patients' office visits. To obtain associated expenditure data, we used the prescribed medicine file of the MEPS, data collected by Agency of Healthcare Research and Quality (AHRQ) for the same study period. The MEPS is the most complete large-scale survey on the use of health care services and pharmaceuticals and associated expenditures in the US. We used the MEPS to estimate expenditures on opioid prescription drugs in US outpatient settings during the study period, as well as patient's out-ofpocket cost for opioids.

\section{Study Sample}

The study sample included ambulatory care visits The study sample included weighted total 126,546 ambulatory care visits made by adult residents in U. S. outpatient physician offices from 2006 to 2010.

\section{Measures}

Opioid prescriptions: The primary dependent variable was an opioid prescribed by a physician during an outpatient visit. Beginning with the 2006 survey year, NAMCS reported drugs prescribed during an ambulatory care visit using Lexicon Plus ${ }^{\circledR}$, a proprietary database of the Cerner Multum, Incorporated [22]. Opioid prescription medications were identified based on the updated guidance provided on the NAMCS website [23]. Then, a binary variable, opioid prescription was coded "yes" if any opioid drug was prescribed during the visit.

Socio-demographics: Socio-demographics included age group (19-34 age group, 35-49, 50-64, 65-79, 80+years), race (White, African American and Other), poverty status ( $>10 \%$ and $>10 \%$ poor in patient's zip code), education attainment $(<20 \%$ and $>20 \%$, college graduates in patient's zip code) primary care visit, physician specialty (medicine/primary care and surgery), health insurance type (private, Medicare/Medicaid and others), cancer diagnosis, median household income in patient's zip code, and injury/poisoning/adverse drug event visit, and time spent with the physician (less than one minute, 1-5 minutes, 6-10 minutes, 11-20 minutes, 21-30 minutes, and more than 30 minutes).

Statistical Analysis: Descriptive statistics were used to identify the number of ambulatory visits that included an opioid prescription and to describe patient's characteristics. Survey weight adjusted serial logistic regression models were fitted to obtain the adjusted odds ratio with all covariates in the model. We used survey weights for all analyses using STATA 13.1 [24]. We also used survey weights for MEPS data for estimating the opioid drug prescription volume and expenditures among the adult population during the study period. The analysis of opioid prescription drug expenditures excluded opioid prescriptions filled in hospitals, hospital pharmacies or pharmacies in other health care settings.

\section{Results}

\section{Patient and visit characteristics}


As shown in (Table 1), prescription opioids, as a percentage of all prescriptions, showed a gradual increase over the 5-year period becoming asymptotic by 2010. Associations were consistent across the study period. The majority of the opioid prescriptions were made for the patients who were aged 50-64 years, white, resided in urban areas and zip code with less poor, more median annual household income and more university graduates, had private insurance coverage, and sought care other than primary care over five years. Primary care physicians and medicine specialists were more likely to prescribe opioids than surgeons. Relatively few patients were diagnosed as cancer patients and had adverse drug events/injury/poisoning. During the study period, 11-20 minutes was the average length of a typical ambulatory visit.

Table 1: Weighted Characteristics of Patients and Visits, National Ambulatory Medical Care Survey (NAMCS) 2006-2010.

\begin{tabular}{|c|c|c|c|c|c|}
\hline Characteristics & $\begin{array}{c}2006 \\
\text { Weighted N } \\
\text { (Weighted \%) }\end{array}$ & $\begin{array}{c}2007 \\
\text { Weighted N } \\
\text { (Weighted \%) }\end{array}$ & $\begin{array}{c}2008 \\
\text { Weighted N } \\
\text { (Weighted \%) }\end{array}$ & $\begin{array}{c}2009 \\
\text { Weighted N } \\
\text { (Weighted \%) }\end{array}$ & $\begin{array}{c}2010 \\
\text { Weighted N } \\
\text { (Weighted \%) }\end{array}$ \\
\hline \multicolumn{6}{|c|}{ \# Opioid Prescriptions } \\
\hline No & $\begin{array}{c}612,339,345 \\
(85.9)\end{array}$ & $\begin{array}{c}675,332,233 \\
(85.5)\end{array}$ & $\begin{array}{c}655,479,874 \\
(84.4)\end{array}$ & $\begin{array}{c}700,067,942 \\
(82.8)\end{array}$ & $\begin{array}{c}669,059,479 \\
(82.9)\end{array}$ \\
\hline Yes & $100,644,665(14.1)$ & $\begin{array}{c}114,710,219 \\
(14.5)\end{array}$ & $\begin{array}{c}121,308,100 \\
(15.6)\end{array}$ & $\begin{array}{c}145,703,895 \\
\quad(17.2)\end{array}$ & $\begin{array}{c}158,931,149 \\
(17.1)\end{array}$ \\
\hline \multicolumn{6}{|c|}{ Age } \\
\hline 19-34 Years & $\begin{array}{c}125,225,658 \\
(17.6)\end{array}$ & $\begin{array}{c}134554479 \\
(17.0)\end{array}$ & $\begin{array}{c}131285894 \\
(16.9)\end{array}$ & $\begin{array}{c}134976438 \\
(16.0)\end{array}$ & $\begin{array}{c}141700402 \\
(17.5)\end{array}$ \\
\hline $35-49$ Years & $\begin{array}{c}169040807 \\
(23.7)\end{array}$ & $\begin{array}{c}180991273 \\
(22.9)\end{array}$ & $\begin{array}{c}169868514 \\
(21.9)\end{array}$ & $\begin{array}{c}188181343 \\
(22.3)\end{array}$ & $\begin{array}{c}175000300 \\
(21.7)\end{array}$ \\
\hline 50-64 Years & $\begin{array}{c}188880092 \\
(26.5)\end{array}$ & $\begin{array}{c}216282348 \\
(27.4)\end{array}$ & $\begin{array}{c}219498474 \\
(28.2)\end{array}$ & $\begin{array}{c}243100521 \\
(28.7)\end{array}$ & $\begin{array}{c}231848274 \\
(28.7)\end{array}$ \\
\hline 65-79 Years & $\begin{array}{c}158683762 \\
(22.2)\end{array}$ & $\begin{array}{c}182593053 \\
(23.1)\end{array}$ & $\begin{array}{c}179285388 \\
(23.1)\end{array}$ & $\begin{array}{c}196493380 \\
(23.2)\end{array}$ & $\begin{array}{c}184802288 \\
(22.9)\end{array}$ \\
\hline $80+$ Years & $\begin{array}{c}71153691 \\
(10.0)\end{array}$ & $\begin{array}{c}75621299 \\
(9.6)\end{array}$ & $\begin{array}{c}76849704 \\
(9.9)\end{array}$ & $\begin{array}{c}83020155 \\
(9.8)\end{array}$ & $\begin{array}{c}74173722 \\
(9.2)\end{array}$ \\
\hline \multicolumn{6}{|c|}{ Race } \\
\hline White & $\begin{array}{c}610994977 \\
(85.7)\end{array}$ & $\begin{array}{c}656831667 \\
(83.1)\end{array}$ & $\begin{array}{c}658708330 \\
(84.8)\end{array}$ & $\begin{array}{c}719116653 \\
(85.0)\end{array}$ & $\begin{array}{c}682827459 \\
(84.6)\end{array}$ \\
\hline African American & $\begin{array}{c}65025725 \\
(9.1)\end{array}$ & $\begin{array}{c}89169080 \\
(11.3)\end{array}$ & $\begin{array}{c}79563875 \\
(10.2)\end{array}$ & $\begin{array}{l}90006401 \\
(10.7)\end{array}$ & $\begin{array}{c}88914054 \\
(11.0)\end{array}$ \\
\hline Others & $\begin{array}{c}36963308 \\
(5.2)\end{array}$ & $\begin{array}{l}44041705 \\
\quad(5.6)\end{array}$ & $\begin{array}{c}38515769 \\
(5.0)\end{array}$ & $\begin{array}{c}36648783 \\
(4.3)\end{array}$ & $\begin{array}{c}35783473 \\
(4.4)\end{array}$ \\
\hline \multicolumn{6}{|c|}{ Location } \\
\hline Rural & $\begin{array}{c}110392407 \\
(16.5)\end{array}$ & $\begin{array}{c}132700452 \\
(17.8)\end{array}$ & $\begin{array}{c}98200409 \\
(13.0)\end{array}$ & $\begin{array}{c}127985941 \\
(15.6)\end{array}$ & $\begin{array}{c}127171037 \\
(16.1)\end{array}$ \\
\hline Urban & $\begin{array}{c}556667937 \\
(83.5)\end{array}$ & $\begin{array}{c}614221668 \\
(82.2)\end{array}$ & $\begin{array}{c}657156788 \\
(87.0)\end{array}$ & $\begin{array}{c}694750430 \\
(84.4)\end{array}$ & $\begin{array}{c}660895601 \\
(83.9)\end{array}$ \\
\hline \multicolumn{6}{|c|}{ Poverty Percentage Patient's ZIP code } \\
\hline$<10 \%$ Poverty Level & $\begin{array}{c}344004107 \\
(52.7)\end{array}$ & $\begin{array}{l}391467556 \\
(53.7)\end{array}$ & $\begin{array}{c}418402539 \\
(56.7)\end{array}$ & $\begin{array}{c}445696709 \\
(55.5)\end{array}$ & $\begin{array}{c}423942741 \\
(55.4)\end{array}$ \\
\hline$\geq 10 \%$ Poverty Level & $\begin{array}{c}308433659 \\
(47.3)\end{array}$ & $\begin{array}{c}336904675 \\
(46.3)\end{array}$ & $\begin{array}{c}319266674 \\
(43.3)\end{array}$ & $\begin{array}{c}357995567 \\
(44.5)\end{array}$ & $\begin{array}{c}341468174 \\
(44.6)\end{array}$ \\
\hline \multicolumn{6}{|c|}{ Median Household Income } \\
\hline $\begin{array}{c}\text { Below Median } \\
(<\$ 40,627)\end{array}$ & $\begin{array}{c}320913131 \\
(49.2)\end{array}$ & $\begin{array}{c}342618030 \\
(47.0)\end{array}$ & $\begin{array}{c}306931496 \\
(41.6)\end{array}$ & $\begin{array}{c}349459435 \\
(43.5)\end{array}$ & $\begin{array}{l}408167283 \\
(42.7)\end{array}$ \\
\hline $\begin{array}{l}\text { Above Median } \\
(\geq \$ 40,627)\end{array}$ & $\begin{array}{c}331524635 \\
(50.8)\end{array}$ & $\begin{array}{c}385811316 \\
(53.0)\end{array}$ & $\begin{array}{c}430836132 \\
(58.4)\end{array}$ & $\begin{array}{c}454270710 \\
(56.5)\end{array}$ & $\begin{array}{c}541378244 \\
(57.3)\end{array}$ \\
\hline
\end{tabular}


Percent Population with bachelor or Higher degree in Patient's Zip code

\begin{tabular}{|c|c|c|c|c|c|}
\hline$<20 \%$ Graduate & $\begin{array}{c}314251706 \\
(48.2)\end{array}$ & $\begin{array}{c}348938668 \\
(47.9)\end{array}$ & $\begin{array}{c}331790204 \\
(45.0)\end{array}$ & $\begin{array}{c}361459024 \\
(45.0)\end{array}$ & $344711422(45.0)$ \\
\hline$\geq 20 \%$ Graduate & $\begin{array}{c}338186060 \\
(51.8)\end{array}$ & $\begin{array}{l}379490678 \\
(52.1)\end{array}$ & $\begin{array}{l}405977424 \\
(55.0)\end{array}$ & $\begin{array}{l}442271121 \\
\quad(55.0)\end{array}$ & $\begin{array}{l}420945635 \\
(55.0)\end{array}$ \\
\hline \multicolumn{6}{|c|}{ Primary Care } \\
\hline No & $\begin{array}{c}392566084 \\
(56.7)\end{array}$ & $\begin{array}{c}436518564 \\
(59.7)\end{array}$ & $\begin{array}{c}410662982 \\
(56.2)\end{array}$ & $\begin{array}{c}459476412 \\
(57.7)\end{array}$ & \multirow{2}{*}{$\begin{array}{c}464722066(60.7) \\
300602806 \\
(39.3)\end{array}$} \\
\hline Yes & $\begin{array}{c}299255496 \\
(43.3)\end{array}$ & $\begin{array}{l}295263531 \\
(40.3)\end{array}$ & $\begin{array}{c}319489862 \\
(43.8)\end{array}$ & $\begin{array}{c}336265963 \\
(42.3)\end{array}$ & \\
\hline \multicolumn{5}{|c|}{ Physician Specialty } & \\
\hline Surgery & $\begin{array}{c}163392336 \\
(22.9)\end{array}$ & $\begin{array}{c}177308368 \\
(22.4)\end{array}$ & $\begin{array}{c}183426672 \\
(23.6)\end{array}$ & $\begin{array}{c}174424386 \\
(20.6)\end{array}$ & $\begin{array}{c}190429631 \\
(24.0)\end{array}$ \\
\hline Private /Medicine & $\begin{array}{l}549591674 \\
\quad(77.1)\end{array}$ & $\begin{array}{c}612734084 \\
(77.6)\end{array}$ & $\begin{array}{c}593361302 \\
(76.4)\end{array}$ & $\begin{array}{l}671347451 \\
(79.4)\end{array}$ & $614095355(76.0)$ \\
\hline \multicolumn{6}{|c|}{ Health Insurer } \\
\hline Private & $\begin{array}{c}351246246 \\
(52.2)\end{array}$ & $\begin{array}{l}395534268 \\
(52.4)\end{array}$ & $\begin{array}{l}415297133 \\
\quad(55.1)\end{array}$ & $\begin{array}{l}441910924 \\
(53.5)\end{array}$ & $416903723(53.1)$ \\
\hline Medicare/Medicaid & $\begin{array}{c}191237763 \\
(28.4)\end{array}$ & $\begin{array}{l}219988766 \\
(29.1)\end{array}$ & $\begin{array}{c}229116847 \\
(30.4)\end{array}$ & $\begin{array}{c}260845662 \\
(31.6)\end{array}$ & $\begin{array}{c}246177154 \\
(31.3)\end{array}$ \\
\hline Others & $\begin{array}{c}130173621 \\
(19.4)\end{array}$ & $\begin{array}{c}139624510 \\
(18.5)\end{array}$ & $\begin{array}{c}109386660 \\
(14.5)\end{array}$ & $\begin{array}{c}122583598 \\
(14.9)\end{array}$ & $122307135(15.6)$ \\
\hline \multicolumn{6}{|c|}{ Cancer Diagnosis } \\
\hline No & $\begin{array}{c}665120309 \\
(93.3)\end{array}$ & $\begin{array}{c}730841198 \\
(92.5)\end{array}$ & $\begin{array}{c}723341633 \\
(93.1)\end{array}$ & $\begin{array}{l}792864209 \\
(93.7)\end{array}$ & $744965913(92.3)$ \\
\hline Yes & $\begin{array}{l}47863701 \\
(6.7)\end{array}$ & $\begin{array}{c}59201254 \\
(7.5)\end{array}$ & $\begin{array}{c}53446341 \\
\quad(6.9)\end{array}$ & $\begin{array}{c}52907628 \\
(6.3)\end{array}$ & $62559073(7.7)$ \\
\hline \multicolumn{6}{|c|}{ Injury/Poisoning/Adverse Drug Events } \\
\hline No & $\begin{array}{c}662999019 \\
(93.8)\end{array}$ & $\begin{array}{c}780859733 \\
(99.0)\end{array}$ & $\begin{array}{c}767527969 \\
(98.8)\end{array}$ & $\begin{array}{c}834527440 \\
(98.9)\end{array}$ & $799839102(99.0)$ \\
\hline Yes & $\begin{array}{l}43936101 \\
(6.2)\end{array}$ & $\begin{array}{c}7797620 \\
(1.0)\end{array}$ & $\begin{array}{c}8949371 \\
(1.2)\end{array}$ & $\begin{array}{c}9098536 \\
(1.1)\end{array}$ & $7685884(1.0)$ \\
\hline \multicolumn{6}{|c|}{ Average Time spent with Physician } \\
\hline Less than a minute & $\begin{array}{c}27266006 \\
(3.8)\end{array}$ & $\begin{array}{c}28850799 \\
(3.7)\end{array}$ & $\begin{array}{c}21379049 \\
(2.8)\end{array}$ & $\begin{array}{c}21567149 \\
(2.6)\end{array}$ & $\begin{array}{c}22859990 \\
(2.8)\end{array}$ \\
\hline 1-10 Minute & $\begin{array}{c}100586430 \\
(14.1)\end{array}$ & $\begin{array}{c}123713259 \\
(15.6)\end{array}$ & $\begin{array}{c}106766418 \\
(13.7)\end{array}$ & $\begin{array}{c}124012015 \\
(14.7)\end{array}$ & $112214743(13.9)$ \\
\hline 11-20 Minute & $\begin{array}{c}381318555 \\
(53.5)\end{array}$ & $\begin{array}{c}444313071 \\
\quad(56.2)\end{array}$ & $\begin{array}{c}435749775 \\
\quad(56.1)\end{array}$ & $\begin{array}{c}447421846 \\
(52.9)\end{array}$ & $427887017(53.0)$ \\
\hline 21-30 Minute & $\begin{array}{c}128476708 \\
(18.0)\end{array}$ & $\begin{array}{c}135609806 \\
(17.2)\end{array}$ & $\begin{array}{c}142953282 \\
(18.4)\end{array}$ & $\begin{array}{c}171112891 \\
(20.2)\end{array}$ & $158577959(19.6)$ \\
\hline $\begin{array}{l}\text { More than } 30 \\
\text { Minute }\end{array}$ & $\begin{array}{c}75336311 \\
(10.6)\end{array}$ & $\begin{array}{c}57555517 \\
(7.3)\end{array}$ & $\begin{array}{c}69939450 \\
(9.0)\end{array}$ & $\begin{array}{c}81657936 \\
(9.6)\end{array}$ & $\begin{array}{c}85985277 \\
(10.7)\end{array}$ \\
\hline
\end{tabular}




\section{Cancer Therapy \& Oncology International Journal}

\section{Prescription Opioid Drugs Explanatory Factors}

Table 2: Logistic regression models odds of opioid prescriptions, National Ambulatory Medical Care Survey (NAMCS) $2006-2010$.

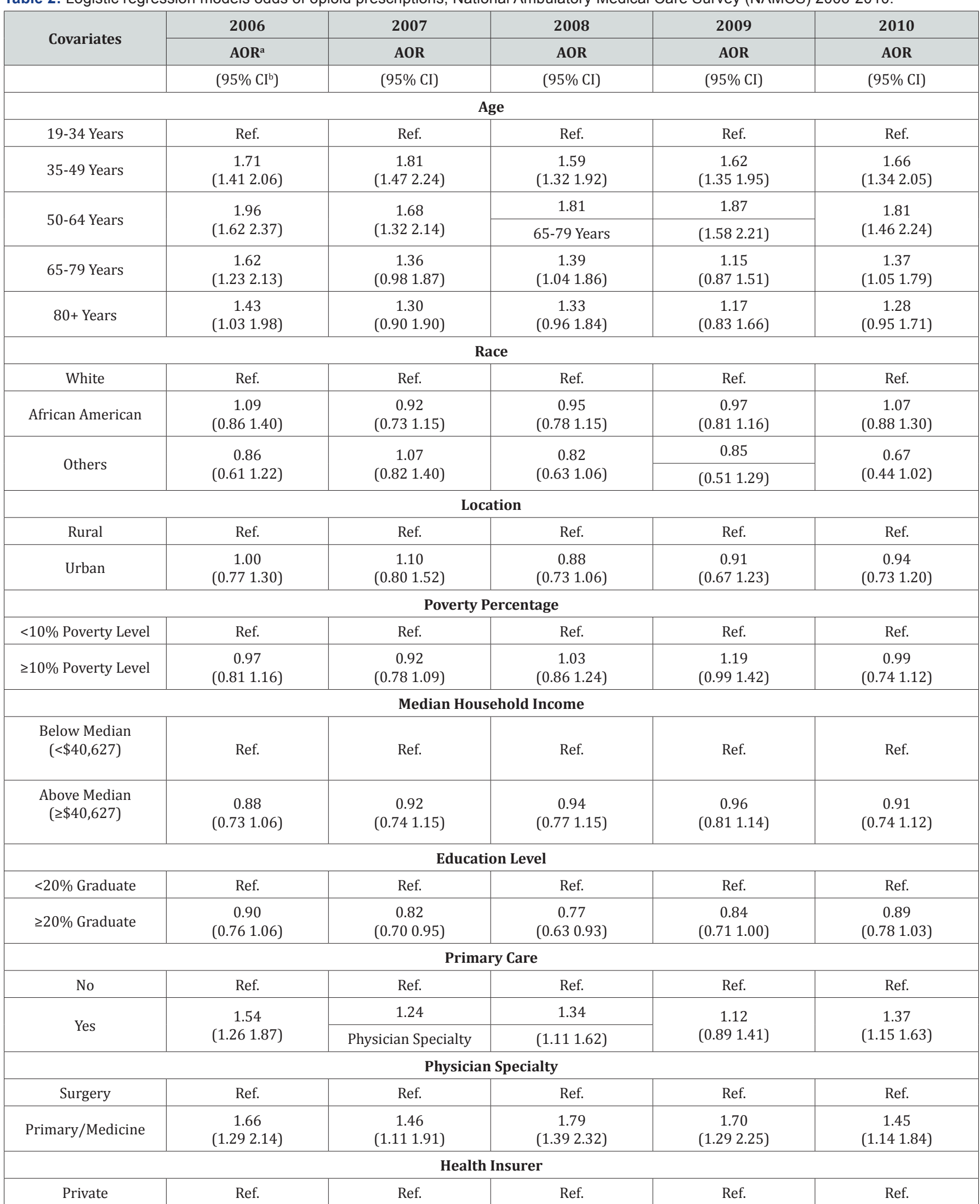




\section{Cancer Therapy \& Oncology International Journal}

\begin{tabular}{|c|c|c|c|c|c|}
\hline Medicare/Medicaid & $\begin{array}{c}1.57 \\
(1.251 .97) \\
\end{array}$ & $\begin{array}{c}1.49 \\
(1.161 .93) \\
\end{array}$ & $\begin{array}{c}1.71 \\
(1.392 .11) \\
\end{array}$ & $\begin{array}{c}1.72 \\
(1.312 .25) \\
\end{array}$ & $\begin{array}{c}1.55 \\
(1.291 .86) \\
\end{array}$ \\
\hline Others & $\begin{array}{c}1.55 \\
(1.271 .88)\end{array}$ & $\begin{array}{c}1.71 \\
(1.412 .08)\end{array}$ & $\begin{array}{c}1.47 \\
(1.231 .76)\end{array}$ & $\begin{array}{c}1.23 \\
(1.011 .51)\end{array}$ & $\begin{array}{c}1.71 \\
(1.402 .08)\end{array}$ \\
\hline \multicolumn{6}{|c|}{ Cancer Diagnosis } \\
\hline No & Ref. & Ref. & Ref. & Ref. & Ref. \\
\hline Yes & $\begin{array}{c}1.17 \\
(0.941 .46)\end{array}$ & $\begin{array}{c}1.03 \\
(0.861 .25)\end{array}$ & $\begin{array}{c}1.27 \\
(1.041 .54)\end{array}$ & $\begin{array}{c}1.11 \\
(0.891 .39)\end{array}$ & $\begin{array}{c}1.27 \\
(1.001 .60)\end{array}$ \\
\hline \multicolumn{6}{|c|}{ Injury/Poisoning/Adverse Drug Events } \\
\hline No & Ref. & Ref. & Ref. & Ref. & Ref. \\
\hline Yes & $\begin{array}{c}1.79 \\
(1.342 .39) \\
\end{array}$ & $\begin{array}{c}1.41 \\
(0.812 .45)\end{array}$ & $\begin{array}{c}1.53 \\
(0.862 .72) \\
\end{array}$ & $\begin{array}{c}1.61 \\
(1.082 .40) \\
\end{array}$ & $\begin{array}{c}1.86 \\
(1.063 .26)\end{array}$ \\
\hline \multicolumn{6}{|c|}{ Time spent with Physician } \\
\hline Less than a minute & Ref. & Ref. & Ref. & Ref. & Ref. \\
\hline 1-10 Minute & $\begin{array}{c}0.95 \\
(0.601 .50) \\
\end{array}$ & $\begin{array}{c}1.30 \\
(0.782 .17) \\
\end{array}$ & $\begin{array}{c}1.11 \\
(0.612 .04) \\
\end{array}$ & $\begin{array}{c}1.65 \\
(0.992 .74) \\
\end{array}$ & $\begin{array}{c}1.27 \\
(0.782 .09) \\
\end{array}$ \\
\hline 11-20 Minute & $\begin{array}{c}1.52 \\
(1.022 .28) \\
\end{array}$ & $\begin{array}{c}1.80 \\
(1.142 .83) \\
\end{array}$ & $\begin{array}{c}1.66 \\
(0.932 .96) \\
\end{array}$ & $\begin{array}{c}2.13 \\
(1.273 .55) \\
\end{array}$ & $\begin{array}{c}1.67 \\
(1.042 .69) \\
\end{array}$ \\
\hline 21-30 Minute & $\begin{array}{c}1.91 \\
(1.222 .97) \\
\end{array}$ & $\begin{array}{c}1.71 \\
(1.072 .73) \\
\end{array}$ & $\begin{array}{c}1.59 \\
(0.922 .74) \\
\end{array}$ & $\begin{array}{c}2.38 \\
(1.404 .04) \\
\end{array}$ & $\begin{array}{c}1.78 \\
(1.102 .88) \\
\end{array}$ \\
\hline More than 30 Minute & $\begin{array}{c}1.96 \\
(1.273 .03)\end{array}$ & $\begin{array}{c}1.59 \\
(0.982 .59)\end{array}$ & $\begin{array}{c}1.62 \\
(0.932 .82)\end{array}$ & $\begin{array}{c}2.11 \\
(1.263 .53)\end{array}$ & $\begin{array}{c}1.45 \\
(0.892 .36)\end{array}$ \\
\hline
\end{tabular}

AOR: Adjusted Odds Ratio; ${ }^{\mathrm{b}} \mathrm{Cl}$ : Confidence Interval

Table 2 reports adjusted odd ratios from logistic regression analyses. Over the five-year study period, patients in the higher age brackets had greater odds of being prescribed an opioid during an ambulatory visit. No statistically significant differences in opioid prescribing were observed by racial group or respondents' area of residence. Poverty status, annual median household income, and educational attainment in the patients' zip code also had no statistically significant association with the opioid prescription drug use. Primary care visits were more likely to result in opioid prescriptions over all five years compared to specialty visits, accordingly, primary care physicians/medicine specialists were more likely to prescribe opioids in all years assessed. Cancer patients also were more likely to be prescribed opioids in 2008 and 2010. For injury/poisoning/adverse drug related events, opioid prescribing was more likely in 2006, 2009, and 2010. Physician visits with longer duration were more likely to result in opioid prescription drug than visits lasting less than one minute.

\section{Expenditures on Opioid Prescription Drugs}

Similar patterns in opioid prescription drug use in U.S. ambulatory settings was observed using MEPS data (Table 3). While the number of opioid prescriptions increased over the study period, the mean total expenditures per opioid prescription decreased from $\$ 151.48$ in 2006 to $\$ 129.31$ in 2010, suggesting a shorter course of therapy per prescription. A similar downward trend was found in the mean of out-of-pocket expenditures per patient ( $\$ 38.57$ in 2006 to $\$ 16.74$ in 2010 ). Total health care expenditures on opioid prescription drugs declined from $\$ 3,720$ million in 2006 to $\$ 2,960$ million in 2008 , but increased from 2009 to 2010 reaching $\$ 3700$ million in 2010 . However, out-ofpocket expenditures on prescription opioids decreased from $\$ 947$ million in 2006 to $\$ 479$ million in 2010.

Table 3: Costs of Prescription Opioids in the Ambulatory Care Settings in the US, Medical Expenditure Panel Survey (MEPS) $2006-2010$.

\begin{tabular}{|c|c|c|c|c|c|}
\hline Variable & $\begin{array}{c}2006 \\
\text { Estimate (SEa) }\end{array}$ & $\begin{array}{c}2007 \\
\text { Estimate (SE) }\end{array}$ & $\begin{array}{c}2008 \\
\text { Estimate (SE) }\end{array}$ & $\begin{array}{c}2009 \\
\text { Estimate (SE) }\end{array}$ & $\begin{array}{c}2010 \\
\text { Estimate (SE) }\end{array}$ \\
\hline $\begin{array}{l}\text { Opioid Prescription filled in } \\
\text { ambulatory settings }\end{array}$ & $\begin{array}{c}24542819 \\
(0.95)\end{array}$ & $\begin{array}{c}23447168 \\
(0.85)\end{array}$ & $\begin{array}{c}24600194 \\
(0.92)\end{array}$ & $\begin{array}{c}28305392 \\
(1.05)\end{array}$ & $\begin{array}{c}28639546 \\
(1.03)\end{array}$ \\
\hline $\begin{array}{l}\text { Mean Total Expenditures per } \\
\text { prescription }\end{array}$ & $\begin{array}{l}\$ 151.48 \\
(42.83)\end{array}$ & $\begin{array}{l}\$ 140.56 \\
(16.15)\end{array}$ & $\begin{array}{l}\$ 120.37 \\
(24.57)\end{array}$ & $\begin{array}{l}\$ 115.16 \\
(10.35)\end{array}$ & $\begin{array}{l}\$ 129.31 \\
(14.98)\end{array}$ \\
\hline
\end{tabular}




\section{Cancer Therapy \& Oncology International Journal}

\begin{tabular}{|c|c|c|c|c|c|}
\hline $\begin{array}{l}\text { Mean Total Out-of-pocket } \\
\text { expenditures per prescription }\end{array}$ & $\begin{array}{l}\$ 38.57 \\
(13.46)\end{array}$ & $\begin{array}{l}\$ 33.15 \\
(7.88)\end{array}$ & $\begin{array}{l}\$ 19.27 \\
(2.50)\end{array}$ & $\begin{array}{l}\$ 19.12 \\
(2.43)\end{array}$ & $\begin{array}{l}\$ 16.74 \\
(1.99)\end{array}$ \\
\hline $\begin{array}{l}\text { Total expenditures of } \\
\text { Prescription opioids }\end{array}$ & $\begin{array}{l}\text { \$3720 Million } \\
\text { (1150 Million) }\end{array}$ & $\begin{array}{l}\text { \$3300 Million } \\
\text { (494 Million) }\end{array}$ & $\begin{array}{l}\$ 2960 \text { Million } \\
\text { (719 Million) }\end{array}$ & $\begin{array}{l}\text { \$3260 Million } \\
\text { (455 Million) }\end{array}$ & $\begin{array}{c}\$ 3700 \\
\text { Million } \\
\text { (527 Million) }\end{array}$ \\
\hline $\begin{array}{c}\text { Total Out-of-pocket } \\
\text { expenditures Prescription } \\
\text { opioids }\end{array}$ & $\begin{array}{l}\text { \$947 Million } \\
\text { (354 Million) }\end{array}$ & $\begin{array}{l}\text { \$777 Million } \\
\text { (205 Million) }\end{array}$ & $\begin{array}{l}\text { \$474 Million } \\
\text { (76.1 Million) }\end{array}$ & $\begin{array}{l}\text { \$541 Million } \\
\text { (91.7 Million) }\end{array}$ & $\begin{array}{l}\text { \$479 Million } \\
\text { (68.6 Million) }\end{array}$ \\
\hline
\end{tabular}

SE: Standard Error

\section{Discussion}

Using 2006-2010 data derive from two nationally representative surveys (i.e., NAMCS and MEPS), this study examined opioid prescribing trends for US adults in outpatient physician offices and related expenditures. Study results showed that opioid prescribing rates increased over the study period and that opioid prescription drug use increased with patient's age. Opioid prescribing was most likely to occur during a primary care visit and physician visit that is longer than 30 minutes.

Trends in opioid prescribing patterns observed in this study, are consistent with other national data sources that quantify opioids prescribed annually in the U.S. However, current evidence suggests that despite the increasing use of opioids, there has been little improvement in chronic pain management and overall health outcomes among Americans [25]. This disparity between opioid drug use and chronic pain management may stem from barriers to cost-effective pain management strategies, including effective regulatory initiatives to contain the escalating opioid misuse [26]. Specific barriers to pain management include limited physician education on safe opioid prescribing, providers' low use of prescription drug monitoring programs (PDMPs), and inconsistent evidence on the effectiveness of PDMPs.

Of note, we found that primary care physicians were more likely to prescribe opioids than surgeons. Prior research suggests that primary care physicians may play a critical role in discerning between appropriate use of opioids and potential misuse and abuse [27]. Recent advances integrating behavioral health care strategies and pharmacological treatment have resulted in primary care physicians' treatment of patients with complex clinical, behavioral and mental health disorders, such as the co-occurrence of chronic clinical conditions with mental health and substance abuse disorders, potentially leading to an increase in prescription opioids. Although these medical advances may improve health-related outcomes, they also place a greater burden on primary care providers not only to identify patterns of behavior and factors that may predispose patients to abuse opioids, but to also manage the prescription drug misuse and abuse. One aspect of this health care utilization burden among primary care physicians is likely the length of outpatient physician visits.
Prior research found that race and ethnicity was associated with differences in use of opioid prescription drugs with Caucasian patients more likely to receive opioid prescriptions compared to racial minority patients [28]. We found no statistically significant differences in opioid prescribing trends among racial population groups. While, African Americans were less likely to be prescribed opioids, compared to Caucasians, (not shown), the difference was not statistically significant.

Opioid prescription drug expenditures showed an upward trend since 2009 after declining for two years (2006-2008). The initial decline in expenditures may be related in part to the Medicare Part D prescription drug plan effective since January 1, 2006. Similar to our study findings, publicly funded health care programs are more likely to cover prescription opioids than private insurers. In addition, we found that elderly populations ( $>=65$ ) were more likely to use opioids than any other age group. Medicare Part D in particular is significantly associated with the out-of-pocket expenses incurred by elderly patients which declined to less than half of what they paid back in 2006. Generic competition may be also behind the lower opioid prescription drug prices which, in turn, explain the decrease in opioid expenditures [29].

The strengths of our study include the use of NAMCS data, one of the largest nationally representative databases on the provision and utilization of outpatient medical care services in the U.S. Using multiple years including the most recently available data, we were able to assess the most recent opioid prescribing trends, which provides an opportunity for informed future prevention strategies. Additionally, we used MEPS, another nationally representative dataset which captures expenditures on healthcare services and prescription drugs. The prescription drug component of the data set is a comprehensive and reliable source for estimating expenditures on prescribed pharmaceuticals.

Despite these strengths, our study has some limitations. Because the NAMCS data do not provide patient-level data, determination of prevalence and incidence was not possible. Additionally, NAMCS is a cross-sectional survey of a random sample of U.S. physician office visits; therefore, we were unable to assess whether the opioid being prescribed was for a patient's chronic pain therapy or acute pain relief. While this was a 


\section{Cancer Therapy \& Oncology International Journal}

limitation affecting our ability to make inferences regarding adherence to best practices in pain management, our findings may lend themselves to generation of hypotheses that could inform future inquiry about medical diagnoses and procedures associated with opioid prescribing and the safety of opioid prescribing patterns in various medical settings.

\section{Acknowledgement}

Authors acknowledge The National Center for Health Statistics (NCHS), a part of the Centers for Disease Control and Prevention (CDC), Department of Health and Human Services (DHS), for providing the NAMCS data used in this study. Authors also acknowledge Mary Jo Larson PhD, MPA for her critical review of a draft of the manuscript. Dr. Larson is a Senior Scientist and Lecturer, Heller School for Social Policy and Management, Brandeis University, Waltham, MA, USA Email: larson@brandeis. edu

\section{Reference}

1. Tsang A, Von Korff M, Lee S, Alonso J, Karam E, et al. (2008) Common chronic pain conditions in developed and developing countries: gender and age differences and comorbidity with depression-anxiety disorders. J Pain 9(10): 883-891.

2. Institute of Medicine (IOM) Committee on Advancing Pain Research C, Education (2011) Relieving Pain in America: A Blueprint for Transforming Prevention, Care, Education, and Research: National Academies Press.

3. Rosenblum A, Marsch LA, Joseph H, Portenoy RK (2008) Opioids and the treatment of chronic pain: controversies, current status, and future directions. Exp Clin Psychopharmacol 16(5): 405-416.

4. US Department of Health and Human Services (HHS) (2011) Results from the 2010 National Survey on Drug Use and Health: Summary of national findings. Substance Abuse and Mental Health Services Administration: Rockville, MD, USA.

5. Centers for Disease Control and Prevention (CDC) (2011) Vital signs: overdoses of prescription opioid pain relievers---United States, 1999-2008. MMWR Morbidity and mortality weekly report 60(43): 1487 1492.

6. Centers for Disease Control and Prevention (CDC) (2010) Unintentional drug poisoning in the United States: CDC Data Brief, July.

7. National Institute on Drug Abuse (NIDA) Prescription Drug Abuse. Research Report Series. Bethesda, MD: National Institute on Drug Abuse (NIDA).

8. Caudill-Slosberg MA, Schwartz LM, Woloshin S (2004) Office visits and analgesic prescriptions for musculoskeletal pain in US: 1980 vs. 2000. Pain 109(3): 514-519.

9. Larochelle MR, Zhang F, Ross-Degnan D, Wharam JF (2015) Trends in opioid prescribing and co-prescribing of sedative hypnotics for acute and chronic musculoskeletal pain: 2001-2010. Pharmacoepidemiol Drug Saf 24(8): 885-892.

10. Wilson RD (2009) Analgesic prescribing for musculoskeletal complaints in the ambulatory care setting after the introduction and withdrawal of cyclooxygenase-2 inhibitors. Arch Phys Med Rehabil 90(7): 1147-1151.

11. Mafi JN, Edwards ST, Pedersen NP, Davis RB, McCarthy EP, et al. (2015) Trends in the Ambulatory Management of Headache: Analysis of
NAMCS and NHAMCS Data 1999-2010. J Gen Intern Med 30(5): 548555 .

12. McDonald DD, Walsh S (2012) Older adult osteoarthritis pain management: results from the 2008 National Ambulatory Medical Care Survey. J Am Acad Nurse Pract 24(2): 107-112.

13. Daubresse M, Chang HY, Yu Y, Viswanathan S, Shah ND, et al. (2013) Ambulatory diagnosis and treatment of nonmalignant pain in the United States, 2000-2010. Med Care 51(10): 870-878.

14. Prunuske JP, Hill CAS, Hager KD, Lemieux AM, Swanoski MT, et al. (2014) Opioid prescribing patterns for non-malignant chronic pain for rural versus non-rural US adults: a population-based study using 2010 NAMCS data. BMC Health Serv Res 14: 563.

15. Sullivan MD, Edlund MJ, Fan MY, DeVries A, Braden JB, et al. (2008) Trends in use of opioids for non-cancer pain conditions 2000-2005 in commercial and Medicaid insurance plans: the TROUP study. Pain 138(2): 440-449.

16. Harle CA, Cook RL, Kinsell HS, Harman JS (2014) Opioid Prescribing by Physicians With and Without Electronic Health Records. J Med Syst 38(11): 138.

17. Kao MC, Minh LC, Huang GY, Mitra R, Smuck M (2014) Trends in Ambulatory Physician Opioid Prescription in the United States, 19972009. PM R 6(7): 575-582.

18. Olsen Y, Daumit GL, Ford DE (2006) Opioid prescriptions by US primary care physicians from 1992 to 2001. J Pain 7(4): 225-235.

19. Kelly JP, Cook SF, Kaufman DW, Anderson T, Rosenberg L, et al. (2008) Prevalence and characteristics of opioid use in the US adult population. Pain 138(3): 507-513.

20. Steinman MA, Komaiko KD, Fung KZ, Ritchie CS (2015) Use of opioids and other analgesics by older adults in the United States, 1999-2010. Pain Med 16(2): 319-327.

21. Centers for Disease Control and Prevention (CDC) Ambulatory Health Care Data.

22. Centers for Disease Control and Prevention (CDC) Trend Analysis Using NAMCS and NHAMCS Drug Data.

23. Centers for Disease Control and Prevention (CDC) The New Ambulatory Care Drug Database System.

24. StataCorp (2013) Stata Statistical Software: Release 13. College Station, TX: Stata Corp LP.

25. Jacox A, Carr D, Payne R (1994) Management of cancer pain. Clinical Practice Guideline Number 9. Rockville, MD: Agency for Health Care Policy and Research, US Dept of Health and Human Services, Public Health Service.

26. Joranson DE, Gilson AM (1998) Regulatory barriers to pain management. Elsevier 14(2): 158-163.

27. Bonhomme J, Shim RS, Gooden R, Tyus D, Rust G (2012) Opioid addiction and abuse in primary care practice: a comparison of methadone and buprenorphine as treatment options. J Natl Med Assoc 104(7-8): 342-350.

28. Pletcher MJ, Kertesz SG, Kohn MA, Gonzales R (2008) Trends in opioid prescribing by race/ethnicity for patients seeking care in US emergency departments. JAMA 299(1): 70-78.

29. Berndt ER, Mortimer R, Bhattacharjya A, Parece A, Tuttle E (2007) Authorized generic drugs, price competition, and consumers' welfare. Health Aff 26(3): 790-799. 
This work is licensed under Creative Commons Attribution 4.0 License DOI: 10.19080/CTOIJ.2017.03.555611

\section{Your next submission with Juniper Publishers} will reach you the below assets

- Quality Editorial service

- Swift Peer Review

- Reprints availability

- E-prints Service

- Manuscript Podcast for convenient understanding

- Global attainment for your research

- Manuscript accessibility in different formats ( Pdf, E-pub, Full Text, Audio)

- Unceasing customer service

Track the below URL for one-step submission https://juniperpublishers.com/online-submission.php 